\title{
Les difficultés de l'autorité et de sa légitimité
}

\section{Jean Martin}

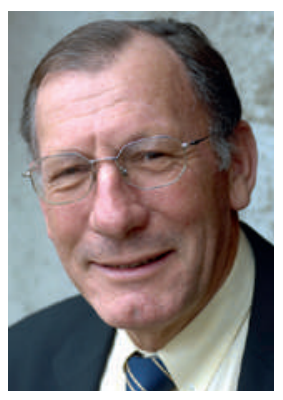

Clairement, ces dernières décennies, l'autorité (de certaines personnes ou institutions) ne va plus de soi, est bousculée voire «aux abonnés absents». Le philosophe Robert Damien consacre un livre à ce sujet [1]. Il insiste sur le sens étymologique d'autorité, à savoir la capacité de s'augmenter, d'être plus et meilleur ensemble. A son avis: «Nous ne vivons pas une crise de l'autorité mais des autorités. En contexte démocratique, il y a une pluralité d'autorités, toutes légitimes, qui définissent nos appartenances, mais qui sont en concurrence. Il faudrait à cet égard arriver à un ‘pluralisme cohérent ‘.» C'est un grand défi: "Comment peut-on être plusieurs tout en restant un? Comment être industriel et écologiste, français - ou suisse - et européen, mondial et national? Nous vivons une crise des idéalisations, des croyances symboliques qui structurent notre histoire.» A cela s'ajoutent deux phénomènes nouveaux, la révolution numérique et le pouvoir médiatique. Ce dernier contribue à faire le lit de la "dictature douce», ou plus manifeste, qu'exerce aujourd'hui l'opinion publique. Une vraie question en Suisse aussi, où le fonds de commerce de certains est la sacralisation de la vox populi, qui ne saurait se tromper... [2]. Dérive qui fait oublier qu'il est risqué de soumettre au suffrage universel qui ne peut s'exprimer qu'en noir et blanc, par oui ou non, des questions dont la complexité et les liens avec d'autres sujets sont tels qu'il est indispensable, pour fonder raisonnablement un avis, de pouvoir s'informer et débattre préalablement, de manière suffisamment complète - ce que peut/doit faire un parlement.

«Notre tradition est antiautoritaire», dit Damien, "où la liberté réside dans la capacité de décider par soimême sans rien devoir à personne. Or, on a confondu l'autorité avec son abus [alors que] il n'y pas d'autorité sans respect ni réciprocité. Quelles que soient la taille et la nature du groupe, il faut que ceux sur qui s'exerce l'autorité aient le sentiment par leur relation de s'élever, de grandir. [...] Comment assumer le déclin des absolus tout en pensant une autorité légitime?» A la question fondamentale de Dostoïevski «Devant qui s'incliner?», Damien répond: «Devant une majorité fraternelle qui me permet de m'augmenter sans léser mes intérêts et en me donnant toujours la liberté de critiquer.»
A propos du rôle de conseiller du prince joué parfois par des philosophes: «Toute personne [même celle en position d'autorité] est un être en devenir équivoque, dans l'incertain, et elle doit pouvoir entendre d'autres voix que la sienne pour s'accomplir. Pour constituer cette autorité légitime, il faut des qualités comme l'intelligence instruite, le discernement, la continuité conséquente. Ces qualités s'acquièrent en rencontrant d'autres points de vue.» Important, à l'évidence.

«Je parle d'une <esth/éthique» de l'autorité, pour dire qu'il n'y a pas d'autorité sans corps augmenté par une puissance symbolique. Quand le corps personnel de la «carcasse` prend le dessus sur le corps symbolique de la fonction, l'autorité fuit dans la caricature du guignol.» Il faut ici, je crois [J.M.], relever de vraies différences de culture politique: la formulation ci-dessus paraît frappée du sceau de la France et de son histoire, d'un fonctionnement qui garde des dimensions monarchiques. Dans notre pays où récemment encore on a vu tel Conseiller fédéral aller à vélo à son bureau et où, dans leurs déplacements officiels, l'accompagnement protecteur dont bénéficient les ministres est plus que modeste, ce «corps symbolique» n'a certainement pas le même poids. Et, jusqu'ici, je ne perçois pas chez nous un affaiblissement marqué de la proximité du citoyen avec ses élus, ni d'ailleurs du respect plutôt convivial qui leur est porté (mais il est vrai que le prestige accordé à la fonction n'est plus le même que celui que j'observais il y a un demi-siècle en milieu terrien vaudois) [3]. Que serait un nouvel esprit politique: «la volonté de penser un nouveau cogito, ce que j'appelle un cogito relationnel d'obligations mutuelles et réciproques qui permettrait de constituer un nouvel idéal du nous. Je m’inscris», dit Damien, «dans la tradition des économies de la coopération compétitive. Ce n'est pas l'individu ni le capital qui est producteur de plus-value mais le nous collectif des coordinations.»

1 Damien R. Eloge de l'autorité. Paris: Armand Colin; 2014. Les citations de cet article sont tirées d'un interview publié dans l'hebdomadaire Marianne, 12 décembre 2014, 70-3.

2 Voir sur ce point l'excellent article du prof. Yves Sandoz: Ce peuple qui a toujours raison. Le Temps (Genève), 17 décembre 2014, p. 10.

3 A propos d'autorité, Bertrand Piccard dans son livre récent Changer d'altitude: «Le but d'une relation devrait toujours être de construire une situation win-win. La seule exception est la situation hiérarchique dans laquelle un chef peut être contraint de donner des ordres [qui peuvent ne pas plaire au subordonné].» 